\title{
Disseminated toxoplasmosis in a patient with non-Hodgkin lymphoma
}

\author{
Herold, M A ; Kühne, R ; Vosberg, M ; Ostheeren-Michaelis, S ; Vogt, P ; Karrer, U
}

\begin{abstract}
Toxoplasmosis is a well-recognized opportunistic disease in HIV-infected individuals that is caused by the reactivation of a previous infection, primarily in the central nervous system, during profound immunodeficiency. Toxoplasmosis has been described more rarely in patients with cancer and chemotherapy. We report a case of a patient with a history of chemotherapy for non-Hodgkin lymphoma who developed pain and progressive paresthesia of the right arm 6 weeks after remission. Relapsing lymphoma was suspected, and steroid and radiation treatment were initiated, but the patient died 5 days later due to multiple organ failure. Autopsy revealed disseminated toxoplasmosis. This case illustrates that toxoplasmosis should be suspected in patients with neoplastic disease, especially lymphomas, who present with unexplained neurologic, pulmonary, or febrile symptoms during or after chemotherapy.
\end{abstract}

DOI: https://doi.org/10.1007/s15010-009-9007-5

Posted at the Zurich Open Repository and Archive, University of Zurich ZORA URL: https://doi.org/10.5167/uzh-28961

Journal Article

Published Version

Originally published at:

Herold, M A; Kühne, R; Vosberg, M; Ostheeren-Michaelis, S; Vogt, P; Karrer, U (2009). Disseminated toxoplasmosis in a patient with non-Hodgkin lymphoma. Infection, 37(6):551-554.

DOI: https://doi.org/10.1007/s15010-009-9007-5 


\title{
Disseminated Toxoplasmosis in a Patient with Non-Hodgkin Lymphoma
}

\author{
M.A. Herold, R. Kühne, M. Vosberg, S. Ostheeren-Michaelis, P. Vogt, U. Karrer
}

\begin{abstract}
Toxoplasmosis is a well-recognized opportunistic disease in HIV-infected individuals that is caused by the reactivation of a previous infection, primarily in the central nervous system, during profound immunodeficiency. Toxoplasmosis has been described more rarely in patients with cancer and chemotherapy. We report a case of a patient with a history of chemotherapy for non-Hodgkin lymphoma who developed pain and progressive paresthesia of the right arm 6 weeks after remission. Relapsing lymphoma was suspected, and steroid and radiation treatment were initiated, but the patient died 5 days later due to multiple organ failure. Autopsy revealed disseminated toxoplasmosis. This case illustrates that toxoplasmosis should be suspected in patients with neoplastic disease, especially lymphomas, who present with unexplained neurologic, pulmonary, or febrile symptoms during or after chemotherapy.
\end{abstract}

Infection 2009; 37: 551-554

DOI 10.1007/s15010-009-9007-5

\section{Introduction}

Toxoplasmosis is a well-recognized opportunistic disease in HIV-infected individuals that is caused by the reactivation of a previous infection, primarily in the CNS, during profound immunodeficiency. Toxoplasmosis has been described more rarely in patients with cancer and chemotherapy. We report a fatal case of disseminated toxoplasmosis in which the patient died 6 weeks after finishing chemotherapy for non-Hodgkin lymphoma (NHL).

\section{Case Presentation}

A 56-year-old woman with a 3-year history of oligosymptomatic chronic lymphocytic leukemia experienced a transformation into a high-grade NHL. Partial remission of the NHL was achieved after six cycles of chemotherapy with cyclophosphamide, doxorubicin, vincristine, prednisone, and rituximab ( $\mathrm{R}-\mathrm{CHOP})$. One year later (January 2007) progressive NHL was treated with fludarabine and cyclophosphamide without rituximab. Prophylactic cotrimoxazole was administered against Pneumocystis jiroveci pneumonia $(\mathrm{PcP})$. After six cycles of second line chemotherapy, clinical and serological remission (tumor marker: soluble IL-2 receptor) was documented (May 2007).

Six weeks later the patient complained of pain in the right arm and subsequently developed progressive paresthesia of the dorsal hand and forearm, including a reduced triceps reflex. The MRI of the spinal cord showed a solitary lesion in the cervical region $(\mathrm{C} 5 / 6)$ with peripheral contrast enhancement and perifocal edema consistent with relapsing lymphoma of the CNS (Figure 1). A simultaneous MRI scan of the brain did not reveal any remarkable abnormalities.

Upon admission, laboratory results were normal, with the exception of an increased lactate dehydrogenase (LDH) level (640 U/l; upper limit 429) and a mild renal dysfunction (serum creatinine level $116 \mu \mathrm{mol} / \mathrm{l})$. Hematologic analyses revealed normocytic anemia (hemoglobin $9.9 \mathrm{~g} / \mathrm{dl}$ ) and moderate lymphopenia $(1,050 / \mu \mathrm{l}$; normal range $1,500-4,000 / \mu \mathrm{l})$. These abnormalities were stable during the last 3 months before admission and were interpreted to be lymphoma- and chemotherapyrelated. At this stage, intramedular abscess formation due to toxoplasmosis or bacterial infection was considered highly unlikely because of the clinical presentation and the administration of cotrimoxazole prophylaxis during chemotherapy. Nevertheless, toxoplasma PCR of the CSF was performed.

With the presumptive diagnosis of relapsing lymphoma of the CNS, steroid treatment with methylpredisolone $1 \mathrm{~g}$ daily iv was initiated. Because of progressive neurologic deficits, radiation treatment with 3 Gy at cervical vertebrae 4-7 was also initiated; 2 days later, the symptoms improved.

On the third day, the patient developed a fever of $39.4{ }^{\circ} \mathrm{C}$ without any focal signs, normal inflammatory parameters (C-reactive protein, leucocytes), and unremarkable chest X-ray and urinalysis. Radiation therapy was continued, and empiric antibiotic treatment with meropenem was started. Despite this measures, the clinical condition deteriorated within 2 days when hypotension, tachycardia, and respiratory failure with bilateral pneumonia developed. The patient was admitted to the intensive care unit (ICU) for pulmonary and circulatory support. Notifi-

\footnotetext{
M.A. Herold (corresponding author), R. Kühne, M. Vosberg, S. Ostheeren-Michaelis, P. Vogt, U. Karrer

Division of Infectious Diseases and Hospital Epidemiology, University Hospital of Zurich, Rämistrasse 100, 8091 Zurich, Switzerland;

Phone: (+41/44) 2553-322, Fax: -291,

e-mail: markus.herold@hin.ch
}

Received: December 23, 2008 - Revision accepted: March 2, 2009

Published online: June 4, 2009 


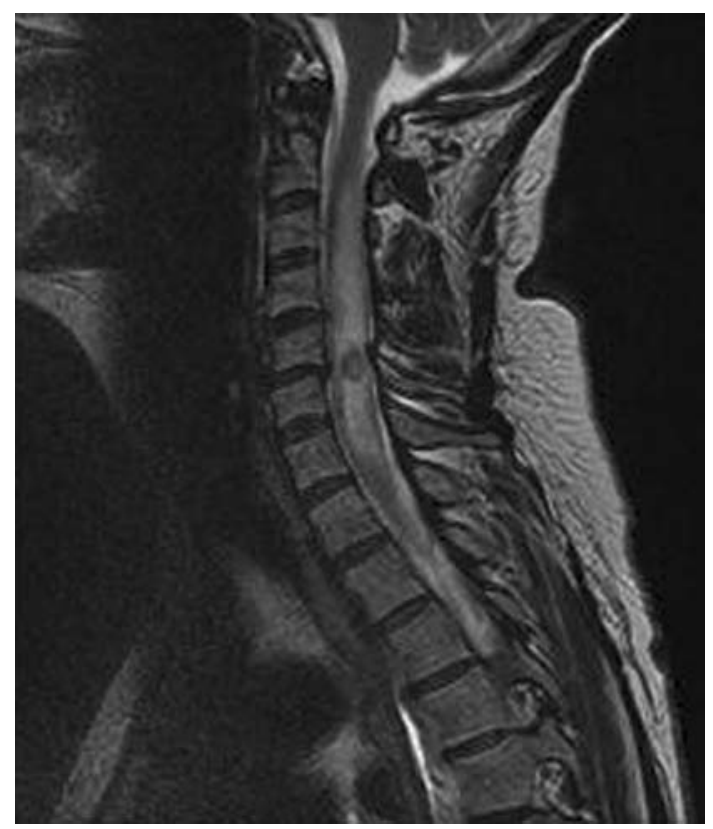

Figure 1. MRI of the spinal cord.

cation of a weakly positive CSF PCR result for Toxoplasma gondii (446 copies/ml; lower limit of detection 300 copies $/ \mathrm{ml}$; quantitative real time PCR, Institute for Medical Microbiology, University of Basel) triggered the initiation of additional treatment with pyrimethamine, sulfadiazine, clindamycine, and folinic acid for toxoplasmosis. However, despite maximal treatment septic shock with multiple organ failure evolved, and the patient died $12 \mathrm{~h}$ after admission into the ICU.

Autopsy and immunohistochemistry revealed disseminated toxoplasmosis with $T$. gondii present in the brain, spinal cord, heart, and lung (Figure 2). The parasite burden was particularly high in the brain and spinal cord where single tachyzoites, but mostly bradyzoites, were detectable in cystic structures in conjunction with nodules of microglia. The lung showed diffuse alveolar damage with edema and congestion. Disseminated areas of necrotizing pneumonia related to toxoplasmosis were mainly evident in the subpleural areas. Myocardial involvement was substantiated by several foci of destructive myocarditis associated with brachyzoites and a cellular infiltrate of lymphocytes. There was no evidence for persistent or recurrent lymphoma in the bone marrow, lymph nodes, brain, or any other organs.

\section{Discussion}

Toxoplasma gondii is a protozoan parasite that infects up to one-third of the world's population. Primary infection is usually clinically silent, but cervical lymphadenopathy or ocular disease may be present in immunocompetent hosts. The parasites usually persist life long in several organs, and reactivation is mainly suppressed by cellular immunity [1]. Consequently, toxoplasma-induced organ disease and death are most prevalent in individuals with severely compromised cellular immunity, for example, with HIV-infection or after transplantation [1]. Reactivation and subsequent pathology is usually confined to a single organ system, and disseminated disease is quite rare but usually fatal in the absence of specific treatment.
Fig. 2. Histology of the patient's autopsy samples: brain (a), lung (b), and heart (c) showing bradyzoites of Toxoplasma gondii (stained with hematoxylin and eosin). d Immunohistochemistry of bone marrow stained for $T$. gondii (polyclonal antibody of rabbit; BioGenex ${ }^{\circledR}$, 1:400; BioGenex Laboratories, San Ramon, CA).
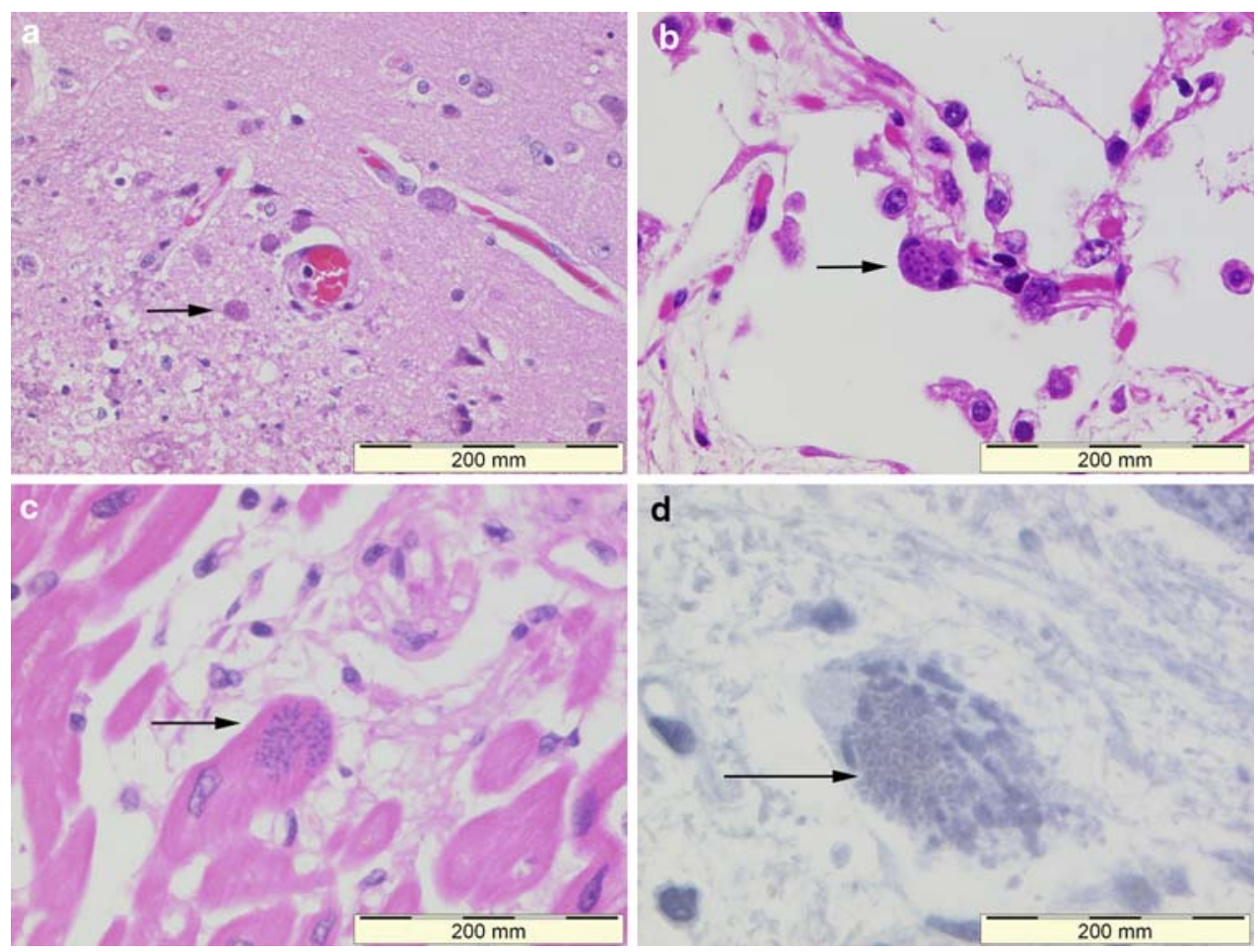
However, several studies, particularly those from the 1970s, have reported cases of disseminated toxoplasmosis during treatment for malignancies, lymphoma, and, in particular, Hodgkin's disease [2-4]. Whether newer treatment regimens for lymphoma or solid malignancies outside the setting of hematopoietic stem cell transplantation (HSCT) carry an increased risk for toxoplasmosis is currently not clear. However, several cases of cerebral and disseminated toxoplasmosis have been reported after fludarabine use $[5,6]$.

Our patient eventually developed rapidly progressive disseminated toxoplasmosis with multiple organ failure due to massive involvement of the lung, heart, and CNS. On admission, there were no signs of heart or lung involvement since creatine kinase (CK) level (81 U/l, upper limit of normal $<167 \mathrm{U} / \mathrm{l})$, the electrocardiogram, and the chest X-ray were unremarkable. Upon ICU admission, unspecific signs of cardiac disease had evolved, the CK levels had risen to $761 \mathrm{U} / \mathrm{l}$, and the ECG showed negative T-waves in I, aVL, and V4-V6. Six weeks after finishing chemotherapy, the patient presented with neurologic signs (brachial paresis) and a solitary medullar lesion that was revealed on the MRI scan. At this time, recurrence of the lymphoma was considered to be the most likely diagnosis. Consequently, radiation and steroid treatment were initiated without attempting a confirmatory biopsy of the lesion due to the substantial risk for neurologic sequelae associated with the procedure. Although the MRI scan was also consistent with toxoplasmosis or bacterial abscess formation, these differential diagnoses were also considered unlikely since cotrimoxazole prophylaxis had been administered until recently and other factors suggestive of bacterial infection were absent.

Retrospectively, events were most probably triggered and accelerated by our stopping the cotrimoxazole prophylaxis after the termination of chemotherapy, unmasking very poor immunocompetence and leading to the first clinical symptom of toxoplasma reactivation in the CNS. The clinical presentation with primarily neurologic signs initially and abscess formation in the CNS strongly suggest that this was a reactivation process and not a primary infection with $T$. gondii. However, since no serological data for toxoplasma are available, primary toxoplasma infection cannot be ruled out completely. Our failure to promptly initiate empiric anti-toxoplasma treatment at initial presentation was fatal in the context of high-dose systemic corticosteroid and local radiation treatment contributing to the fulminant disease course. Specific treatment was initiated too late to effectively treat the disseminated disease.

Toxoplasmosis in the setting of solid tumors and lymphoma and of their treatment is rare, with estimates ranging from $0.02 \%$ in breast cancer up to $3 \%$ in patients with lymphoblastic leukemia [7]. In a review of 128 cases of cancer-associated toxoplasmosis, Israelski and Rem- ington recorded 59 patients with Hodgkin lymphomas, 12 with non-Hodgkin lymphomas, 34 with miscellaneous lymphatic neoplastic diseases, and only 13 with solid tumors [7]. Most cases of toxoplasmosis occurred during progressive neoplastic disease (only 10\% were in stable remission) and patients were receiving corticosteroids, alkylating agents, antimetabolites, and/or radiation therapy. Nearly every organ system was involved, particularly the CNS, heart, and lung.

Our case illustrates that toxoplasmosis should be suspected-if its diagnosis cannot be excluded - aggressively diagnosed, and treated empirically in patients with neoplastic disease, especially lymphomas, who present with unexplained neurologic, pulmonary, or febrile symptoms during or after chemotherapy. This is particularly relevant for immunosuppressed individuals presenting with solitary or multiple CNS lesions. Cotrimoxazole prophylaxis for $\mathrm{PcP}$ is also effective for toxoplasmosis, but breakthrough disease cannot be fully excluded. Toxoplasma serology before the initiation of chemotherapy may be helpful for risk assessment, whereas a specific PCR analysis on the CSF is mainly useful if positive, since the positive predictive value $(90-100 \%)$ is more reliable than the negative predictive value $(70-80 \%)$ [8-10]. A PCR analysis on the peripheral blood was not performed in our patient, but such an analysis may be helpful and has been used in studies of HSCT. However, its diagnostic performance in cancer patients has not yet been sufficiently validated [11].

\section{References}

1. Montoya JG, Liesenfeld O: Toxoplasmosis. Lancet 2004; 363: 1965-1976.

2. Gleason TH, Hamlin WB: Disseminated toxoplasmosis in the compromised host. A report of five cases. Arch Intern Med 1974; 134: 1059-1062.

3. Vietzke WM, Gelderman AH, Grimley PM, Valsamis MP: Toxoplasmosis complicating malignancy. Experience at the National Cancer Institute. Cancer 1968; 21: 816-827.

4. Carey RM, Kimball AC, Armstrong D, Lieberman PH: Toxoplasmosis. Clinical experiences in a cancer hospital. Am J Med 1973; 54: 30-38.

5. Foss FM, Ihde DC, Linnoila IR, Fischmann AB, Schechter GP, Cotelingam JD, Steinberg SM, Ghosh BC, Stocker JL, Bastian A, et al. Phase II trial of fludarabine phosphate and interferon alfa$2 \mathrm{a}$ in advanced mycosis fungoides/Sezary syndrome. J Clin Oncol 1994; 12: 2051-2059.

6. Bacchu S, Fegan C, Neal J: Cerebral toxoplasmosis in a patient with chronic lymphocytic leukaemia treated with fludarabine. Br J Haematol 2007; 139: 349.

7. Israelski DM, Remington JS: Toxoplasmosis in patients with cancer. Clin Infect Dis 1993; 17: S423-S435.

8. Dupon M, Cazenave J, Pellegrin JL, Ragnaud JM, Cheyrou A, Fischer I, Leng B, Lacut JY: Detection of Toxoplasma gondii by PCR and tissue culture in cerebrospinal fluid and blood of human immunodeficiency virus-seropositive patients. J Clin Microbiol 1995; 33: 2421-2426. 
9. Vidal JE, Colombo FA, de Oliveira AC, Focaccia R, Pereira-Chioccola VL: PCR assay using cerebrospinal fluid for diagnosis of cerebral toxoplasmosis in Brazilian AIDS patients. J Clin Microbiol 2004; 42: 4765-4768.

10. Goto M, Takahashi T, Kanda T, Iwamoto A: Detection of Toxoplasma gondii by polymerase chain reaction in cerebrospinal fluid from human immunodeficiency virus-1-infected Japanese patients with focal neurological signs. J Int Med Res 2004; 32: 665-670.
11. Adurthi S, Sahoo T, Chakka K, Radhika B, Appaji L, Bapsy P, Ramesh C, Jayshree R: Acute toxoplasmosis in nonstem cell transplant patients with haematological malignancies: a study from a regional cancer institute in South India. Hematol Oncol 2008; 26: 229-233. 